\title{
Editorial
}

\section{Mathematical Methods Applied to the Celestial Mechanics of Artificial Satellites}

\author{
Antonio F. Bertachini A. Prado, ${ }^{1}$ Maria Cecilia Zanardii, ${ }^{2}$ \\ Tadashi Yokoyama, ${ }^{3}$ Silvia Maria Giuliatti Winter, ${ }^{2}$ \\ and Josep J. Masdemont ${ }^{4}$ \\ ${ }^{1}$ INPE-DMC, Avenida dos Astronautas 1758, 12227-010 São Jose dos Campos, SP, Brazil \\ ${ }^{2}$ Universidade Estadual Paulista (UNESP), Campus de Guaratinguetá, CEP 12516-410 Guaratinguetá, \\ SP, Brazil \\ ${ }^{3}$ Universidade Estadual Paulista, Campus de Guaratinguetá, Caixa Postal 178, 13500-970 Rio Claro, \\ SP, Brazil \\ ${ }^{4}$ IEEC and Departament de Matemàtica Aplicada I, Universitat Politècnica de Catalunya, Diagonal 647, \\ 08028 Barcelona, Spain
}

Correspondence should be addressed to Antonio F. Bertachini A. Prado, prado@dem.inpe.br

Received 9 May 2012; Accepted 9 May 2012

Copyright (c) 2012 Antonio F. Bertachini A. Prado et al. This is an open access article distributed under the Creative Commons Attribution License, which permits unrestricted use, distribution, and reproduction in any medium, provided the original work is properly cited.

Celestial mechanics is a science that comes from the more general field of astronomy. It is devoted to the study of the motion of the planets, moons, asteroids, comets, and other celestial bodies. Some of the main researchers involved in this field are well-known names in history, like Johannes Kepler and Isaac Newton, who wrote the basic laws that govern these motions.

With the advances of technology, the world entered the so-called "Space Age," where artificial artifacts started to be built and launched into space. To perform those tasks, the same laws used to describe the motion of the natural celestial bodies can be used to study the motion of those manmade spacecrafts. Although it is not the beginning of the space activities, the launch of the satellite Sputnik in 1957, by the former Soviet Union, is an important mark of this age. This launch was then followed by the United States of America (USA) that, among other space activities, landed a man on the Moon in 1969.

Nowadays, after those first historical events, the activities related to artificial satellites are one of the most important fields in science and technology. It includes several important applications of engineering that improved the life of everybody in the entire world. Communications by satellite are well established for many years, and it is hard to imagine a world without this capability. Another field that has been helped by the space activities is the field related to the exploration of resources on Earth. Images from satellites can generate important maps to find their locations, and this can make the difference for a sustainable use of our resources. The use of location devices based on the GPS constellation is also everyday 
more common. Several other similar services will be soon provided by Europe, Russia, and China. The applications of this type of service include the monitoring of valuables under transport and the search for the fast way to reach a destination. Artificial satellites are also used to make weather forecast, which can contribute to the very important task of natural disaster prediction. It is not only possible to help agricultural development by predicting the behavior of the weather, but it is also possible to anticipate large-scale phenomenon such as the occurrence of tsunamis. These activities is extremely important for everybody on Earth.

The "Mathematical Methods Applied to the Celestial Mechanics of Artificial Satellites," the title of the present issue of the journal, has a key role in those activities. One of the tasks to be performed when planning a satellite mission is to find the most adequate orbit to place this satellite. After this first step, it is also necessary to study practical problems related to the maintenance of its nominal orbit and how much and when it will be modified by the natural forces present in nature. These questions can be addressed by analyzing the position of the spacecraft. There are also several questions regarding the orientation of the spacecraft. It is necessary to know its orientation and how to control it in order to plan the space missions. Those topics are all covered in the list of papers published in the present volume.

In that scope, this special issue is focused on the recent advances in mathematical methods applied to the celestial mechanics of artificial satellites. It has a total of twenty two papers briefly described below.

Eight of them are concerned with the problems of optimal maneuvers. "Low-thrust orbital transfers in the two-body problem" is written by A. A. Sukhanov and A. F. B. A. Prado. Although several methods for optimization of multirevolution orbital transfers are available in the literature, most of them are rather complicated, with limited usage due to a variety of assumed hypothesis. In this paper, based on a convenient linearization near a reference orbit, the authors propose a very simple method for low-thrust transfers (power limited). The method is free from any averaging concepts, and no limit on the number of orbits around the attracting center nor any constraint on the thrust direction is assumed. Several additional advantages are outlined in the paper.

"Four-impulsive rendezvous maneuvers for spacecraft in circular orbits using genetic algorithms" is written by D. P. S. dos Santos et al.. In this paper, the problem of rendezvous is considered, which is necessary to transfer a spacecraft from one orbit to another, but with the extra constraint of meeting another spacecraft when reaching the final orbit. This work analyzes optimal rendezvous maneuvers between two coplanar circular orbits, seeking to perform this transfer with low fuel consumption, by assuming a time-free problem and using four burns during the process. The genetic algorithm represents a new alternative that can be used for comparisons with the results obtained by standard procedures available in the literature. The results show that this technique brings good results for the proposed fourimpulsive rendezvous maneuvers, when compared with the ones obtained by the traditional impulsive method. Therefore, this approach can be used in real cases, especially when a biimpulsive transfer is not possible due to the limitations of the engine of the spacecraft.

Another paper concerned with the spacecraft maneuvers is "Low-thrust out-of-plane orbital station-keeping maneuvers for satellites," written by V. M. Gomes and A. F. B. A. Prado. This paper considers the problem of-out-of plane orbital maneuvers for station keeping of satellites. The main idea is to consider an Earth satellite in a disturbed orbit, and it is necessary to perform a small amplitude orbit correction to return the satellite to the nominal orbit, to keep it performing its mission. Optimal control is used to build an algorithm to search for solutions for the problem of minimum fuel consumption to make the required orbital 
maneuvers. The problem takes into account the accuracy in the constraint's satisfaction. The adjustments made in the algorithm and the used parameters allow the convergence in most of the analyzed cases. The results show that it is possible to reduce the costs by exploring tolerable errors in the constraint's satisfaction. It can also be observed that the increasing of the number of propulsion arcs decreases the fuel costs. The reason for this fact is that increasing this number causes an increase in the degree of freedom available for the optimization technique. Since the maneuvers have small amplitudes, this increase in the burning arcs has a limit in the savings, so the fuel consumption reaches constant values after a certain value of the number of arcs.

"An adaptive remeshing procedure for proximity maneuvering spacecraft formations" is written by L. Garcia-Taberner and J. J. Masdemont. This paper searches for an optimal way to obtain reconfigurations of spacecraft formations. It uses a discretization of the time interval and obtains local solutions by applying variational method. Applications are made for libration point orbits, trying to find optimal meshes using an adaptive remeshing procedure and on the determination of the parameter that governs it.

Next, "Closed relative trajectories for formation flying with single-input control" is written by A. Guerman et al.. The authors study the problem of formation shape control under a constrained thrust direction. It is considered a formation with two satellites and a linear model for the relative motion (including J2) is used. It is also assumed that the satellites are equipped with a passive attitude control system that provides one-axis stabilization. The propulsion system consists of one or two thrusters oriented along the stabilized axis. Based on those assumptions, the authors prove that, in both cases (passive magnetic attitude stabilization and spin stabilization) and for all initial relative positions and velocities of the satellites, there are controls that guaranty their periodic relative motion.

Another paper of this topic is "Modified Chebyshev-Picard iteration methods for stationkeeping of translunar halo orbits," written by X. Bai and J. L. Junkins. The authors present a new modified Chebyshev-Picard iteration method for the station-keeping of a halo orbit around the $\mathrm{L}_{2}$ libration point of the Earth-Moon system. Compared to other competing methods, the authors emphasize some advantages of the present technique: it does not require weight turning and computation of the state transition matrix so that, computationally, it is clearly very efficient. Also, the final results are given in an orthogonal polynomial form.

Then, the paper "A numerical study of optimal low-thrust limited power trajectories between coplanar circular orbits in an inverse-square force field" is written by S. Fernandes et al.. Motivated by the use of the low-thrust propulsion system in space missions, S. Fernandes et al. presented a numerical analysis of optimal low-thrust limited power trajectories for simple transfers between circular coplanar orbits in an inverse square force field. In this paper, two powerlimited variable ejection velocity systems are analyzed by considering the fuel consumption as the performance criteria. The numerical results showed a good agreement with the analytical results derived from the linear theory. Therefore, the linear theory can be used as a good approximation for the transfer problem with small amplitudes.

Another paper of this sequence is "Optimal two-impulse trajectories with moderate flight time for earth-moon missions," written by S. S. Fernandes and C. M. P. Marinho. The paper considers two-impulse trajectories with moderate flight time for Earth-Moon missions where the goal is to optimize the fuel consumption, expressed by the total characteristic velocity. The well-known patched-conic approximation is used as the dynamical model. After that, two versions of the planar circular restricted three-body problem are also considered. Two parameters are optimized in the two-body version: the initial phase angle of the space vehicle 
and the first impulse. When considering the three-body dynamics, the parameters to be optimized are four: initial phase angle of the space vehicle, the flight time, and the first and the second impulses.

Another topic considered in this issue is related to the determination, simulation, and propagation of trajectories of spacecraft under several different circumstances. Concerning this topic is the paper "Analysis of filtering methods for satellite autonomous orbit determination using celestial and geomagnetic measurement," written by X. Ning et al.. Orbit determination of a satellite is a complex process which evolves the precise estimation of the ephemeris of the satellite. In the paper by Xiaolin et al., the performance of three filters methods, extended Kalman filter (EKF), unscented Kalman filter (UKF), and unscented particle filter (UPF), is analyzed under different conditions. Their simulation results showed that the UPF performs the best while the EKF gives the worst orbit determination (OD) accuracy under the same conditions. The computation cost analysis demonstrates that the UPF-based OD system provides the best orbit determination accuracy.

Another paper of this topic is "The orbital dynamics of synchronous satellites: irregular motions in the $2: 1$ resonance," written by J. C. Sampaio et al.. It analyses the dynamical behavior of a synchronous satellite trapped in a 2:1 resonance with the Earth under the effects of the zonal, $\mathrm{J}_{20}$ and $\mathrm{J}_{40}$ coefficients, and the tesseral harmonics, $\mathrm{J}_{22}$ and $\mathrm{J}_{42}$ coefficients. Through a sample of Mathieu transformations, the order of the dynamical system was reduced. Three critical angles associated to the $2: 1$ resonance were analyzed by numerically simulating the reduced system. Their results showed that for several different values of the initial conditions the system presented a chaotic motion, which was confirmed by calculating the Lyapunov exponents.

Another one in this category is "Application of periapse maps for the design of trajectories near the smaller primary in multi-body regimes," written by K. C. Howell et al.. This paper has the goal of incorporating multibody dynamics into the preliminary design of a mission. This technique adds flexibility to the problem and, in some cases, generates impact in the cost of the maneuver. Attention is given on the development and application of design tools to help preliminary trajectory design considering a multibody environment. Using the model given by the circular restricted three-body problem, a trajectory near the smaller primary is studied. Periapse Poincaré maps are then used to predict both short- and long-term trajectory behaviors. Several trajectories are computed using this approach.

The next paper is entitled "Comparison between two methods to calculate the transition matrix of orbit motion," written by A. P. M. Chiaradia et al.. The goal of this paper is to compare and choose the most suited method to calculate the transition matrix used to propagate the covariance matrix of the position and velocity of the state estimator, within the procedure of the artificial orbit determination. Two methods are evaluated according to accuracy, processing time, and handing complexity of the equations for both circular and elliptical orbits. The first method is an approximation of the Keplerian motion, providing an analytical solution which is calculated numerically by solving the Kepler equation. It is also optimized for an elliptical orbit. The second one is a local numerical approximation that includes the effect of the Earth oblateness; it has no singularity and no restriction about the kind of orbit. By analyzing the results, it is possible to observe that for short periods of time, and when more accuracy is necessary, it is recommended the use of the second method, since the $\mathrm{CP}$ time does not overload excessively the computer during the orbit determination procedure. However, for large intervals of time and when one expects more stability on the calculations, the use of the first method is recommended. 
Another paper of this category is "Semianalytic integration of high-altitude orbits under lunisolar effects," written by M. Lara et al.. In this paper, the long-term behavior of highaltitude orbits with lunisolar perturbations and about a noncentral Earth (including J2 and J3 potential terms) is approached in a semianalytical way using canonical perturbation theory. The authors take advantage of the different scales of time to do the averaging in the extended phase space. In addition, up to second-order terms in the Earth's oblateness coefficient, the averaging has been computed in closed form of the eccentricity, and therefore, the semianalytical integration can be applied to an orbit of arbitrary eccentricity.

The next set of papers is related to the attitude of the satellites, including maneuvering, determination, and propagation of attitude. A paper related to that is "Analysis of attitude determination methods using GPS carrier phase measurements," written by L. Baroni and H. K. Kuga. Global navigation satellite systems (GNSS) can be used to determine the orientation of a platform. By using at least three GPS antennas, properly mounted on a platform, the determination of the baseline vectors, formed between the antennas, is the prerequisite for the attitude determination of this platform. L. Baroni and H. K. Kuga implemented and tested two algorithms (LSAT and LAMBDA) in order to obtain an accurate attitude determination in real time, by using data provided by the GPS receivers. As a result, they found that although both algorithms had a similar performance, the processing time of the LSAT method was larger. Although the inclusion of the quaternions increases the processing time, they are necessary to avoid singularities.

Another one of this topic is "Numerical analysis of constrained, time-optimal satellite reorientation," written by R. G. Melton. The author studies on time-optimal satellite slewing maneuvers with one satellite axis required to obey multiple path constraints. The paper considers four cases in which this axis is either forced to follow a finite arc of the constraint boundary or has initial and final directions that lie on the constraint boundary. It is shown that the precession created by the control torques, moving the axis away from the constraint boundary, provides faster slewing maneuvers. The paper also proposes a two-stage process for the generation of optimal on-board solutions in less time.

Another one is the paper "Study of stability of rotational motion of spacecraft with canonical variables," written by W. R. Silva et al.. In this work, the main problem of the stability of the rotational motion of a satellite is revisited. The most adequate set of variables (Andoyer canonical set) and Kovalev-Savchenko theorem are adopted to analyze the stability of the equilibrium points. Some important optimization on the techniques involved (determination of the equilibrium points, normalization and application of Kovalev-Savchenko theorem) is obtained. Examples considering real satellites with very large number of equilibrium points are analyzed.

Then, the paper "Unscented Kalman filter applied to the spacecraft attitude estimation with Euler angles" is written by R. V. Garcia et al.. In this paper, an algorithm is used to make realtime estimation of the attitude of an artificial satellite. Some real data obtained by the CBERS2 satellite (China-Brazil Earth Resources Satellite) is used. The estimator used for attitude determination is the unscented kalman filter, which is a new alternative to the extended Kalman filter. This algorithm is capable of carrying out estimation of the states of nonlinear systems, and it does not require the linearization of the functions present in the model. It uses a transformation to generate a set of vectors that, after a nonlinear transformation, preserves the same mean and covariance of the random variables obtained before the transformation. A comparison between the unscented Kalman filter and the extended Kalman filter is performed using real data. 
Another of this category is the paper "Higher-order analytical attitude propagation of an oblate rigid body under gravity-gradient torque," written by J. F. San-Juan et al.. This paper is related to the rotation of an oblate rigid body under gravity-gradient torque, a nonintegrable problem that may be analyzed through the perturbation theory. The Andoyer variables, used to describe the rotational motion, simplify the solution of the partial differential equations that provide the generating function of the Lie transforms. It is shown that when a higherorder perturbation theory is used to study the rotation of a uniaxial satellite under gravitygradient torque, this shows that known special configurations of the attitude dynamics at which the satellite rotates, on average, as in a torque-free state, are only the result of an early truncation of the secular frequencies of motion. The higher order of the analytical solution can be compared to the long-term numerical integration of the equations of motion.

Other four papers are related to specific applications, including error analysis of space missions, locations of platforms, and solutions of mathematical equations. One of this type is the paper "Error modeling and analysis for InSAR spatial baseline determination of satellite formation flying," written by J. Tu et al.. Close formation flying satellites equipped with synthetic aperture radar (SAR) antenna have become the focus of space technology. The analysis of the errors introduced by spatial baseline measurement is important for the performance of the SAR satellite system. J. Tu et al. carefully analyzed these errors and classified them into two groups: the errors related to the baseline transformation and those related to the GPS measurements. Through a set of simulations, they verified that the errors related to the GPS measurements are the principal influence on the space baseline determination, while the carrier phase noise of the GPS observation and fixing error of the GPS receiver antenna are the main factors of the errors related to the GPS measurements.

Another one is "IMU fault detection based on X2-CUSUM," written by É. J. de Oliveira et al.. Combining chi-square cumulative sum and media filter techniques, an efficient algorithm is presented for the problem involving fault detection and isolation on inertial measurement units. In particular, the success and feasibility of the method is clearly demonstrated in the case of low-level step fault.

The paper "Assessment of the ionospheric and tropospheric effects in location errors of data collection platforms in equatorial region during high and low solar activity periods," written by Á. A. da Silva et al., provides an assessment of ionospheric effects using IRI (international reference ionosphere) and IONEX (IONosphere map exchange) models and tropospheric delay compensation using climatic data provided by the National Climatic Data CenterNOAA Satellite and Information Service. Two selected DCPs (Data Collection System) are used in conjunction with the Brazilian Data Collection Satellite (SCD2) during high- and low-solar activity periods. Results show that the ionospheric effects on transmission delays are significant in equatorial region and should be considered to reduce the DCP location errors, mainly in high solar activity periods. It is also shown that the platform location errors can be reduced when the ionospheric and the tropospheric effects are properly considered.

Another is the paper "Application of the Hori method in the theory of nonlinear oscillations," written by S. S. Fernandes. In this paper, the Hori method is applied to the theory of nonlinear oscillations. Two algorithms to determine and generate functions and the new system of differential equations are derived, based on a more general algorithm proposed by Sessin (1983). The solutions are not uniquely determined because the algorithms involve arbitrary functions. Different choices of them can be made to simplify the new system of differential equations and to define near-identity transformations. These algorithms are then applied to determine second-order asymptotic solutions of two well-known equations in the theory of nonlinear oscillations: van der Pol and Duffing equations. 


\section{Acknowledgments}

The Guest Editors would like to thanks all the reviewers, the authors, and all the staff of this journal involved in the preparation of this special issue for the opportunity to publish the papers related to this topic.

Antonio F. Bertachini

A. Prado

Maria Cecilia Zanardi

Tadashi Yokoyama

Silvia Maria Giuliatti Winter

Joseph J. Masdemont 


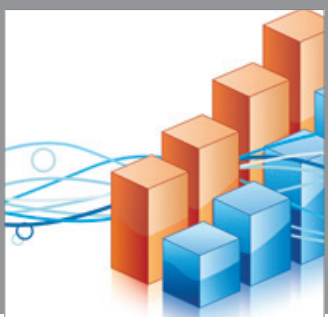

Advances in

Operations Research

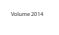

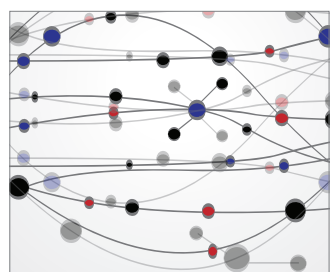

\section{The Scientific} World Journal
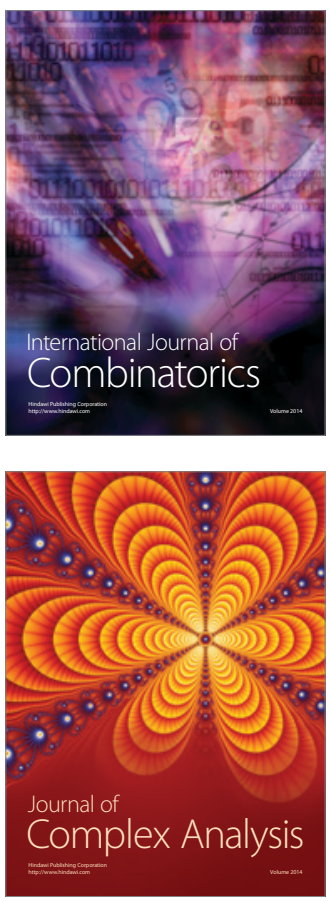

International Journal of

Mathematics and

Mathematical

Sciences
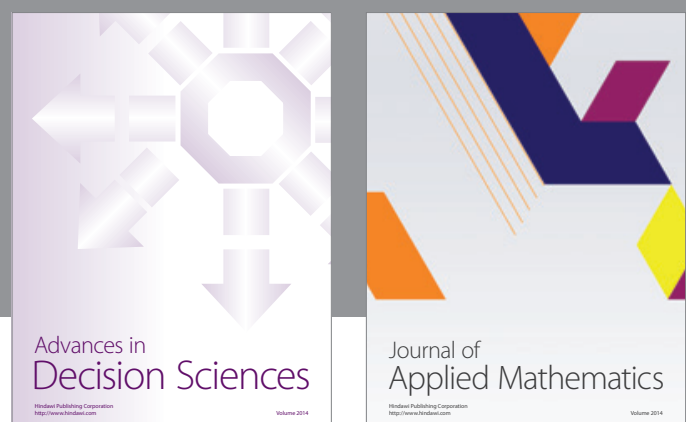

Journal of

Applied Mathematics
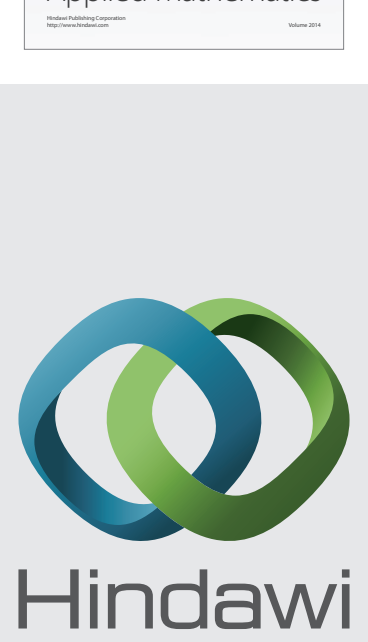

Submit your manuscripts at http://www.hindawi.com
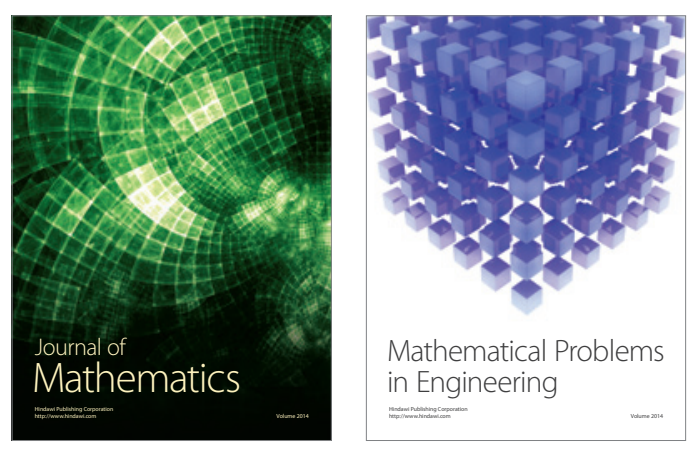

Mathematical Problems in Engineering
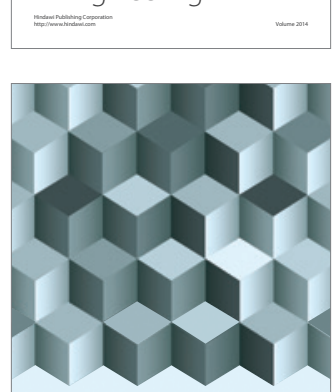

Journal of

Function Spaces
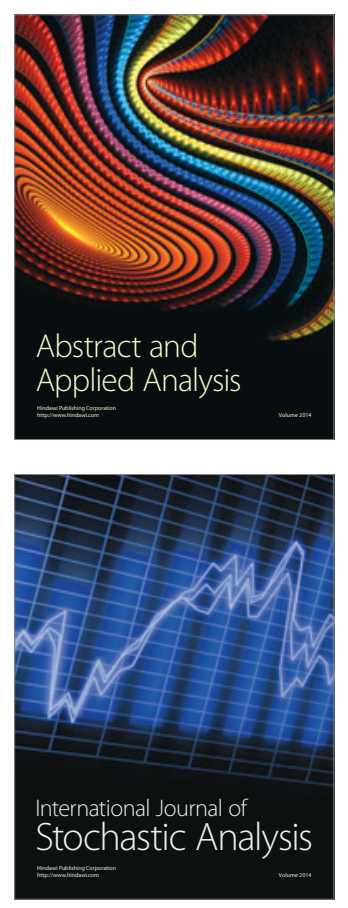

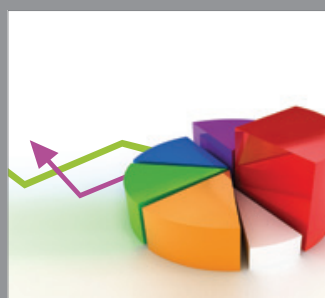

ournal of

Probability and Statistics

Promensencen
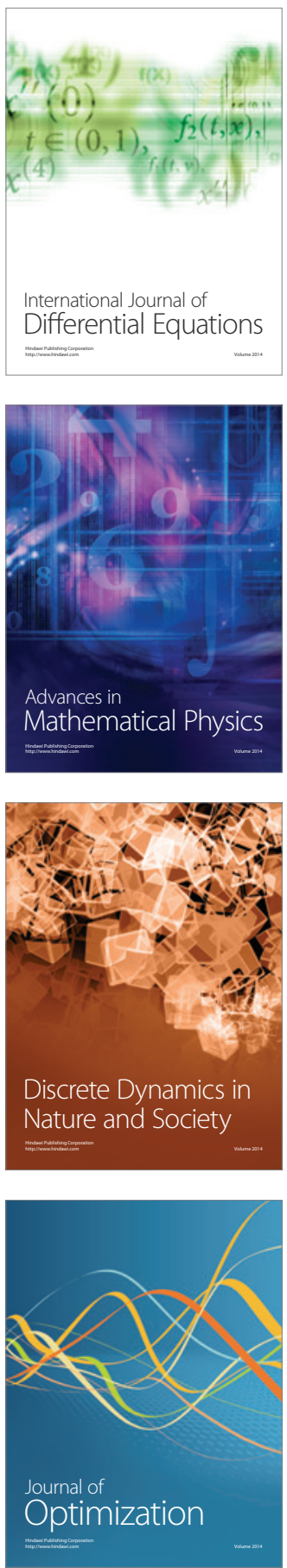